\title{
Fast Estimation of L1-Regularized Linear Models in the Mass-Univariate Setting
}

\author{
Holger Mohr ${ }^{1} \cdot$ Hannes Ruge ${ }^{1}$ \\ Published online: 15 September 2020 \\ (C) The Author(s) 2020
}

\begin{abstract}
In certain modeling approaches, activation analyses of task-based fMRI data can involve a relatively large number of predictors. For example, in the encoding model approach, complex stimuli are represented in a high-dimensional feature space, resulting in design matrices with many predictors. Similarly, single-trial models and finite impulse response models may also encompass a large number of predictors. In settings where only few of those predictors are expected to be informative, a sparse model fit can be obtained via L1-regularization. However, estimating L1-regularized models requires an iterative fitting procedure, which considerably increases computation time compared to estimating unregularized or L2-regularized models, and complicates the application of L1-regularization on whole-brain data and large sample sizes. Here we provide several functions for estimating L1-regularized models that are optimized for the mass-univariate analysis approach. The package includes a parallel implementation of the coordinate descent algorithm for CPU-only systems and two implementations of the alternating direction method of multipliers algorithm requiring a GPU device. While the core algorithms are implemented in C++/CUDA, data input/output and parameter settings can be conveniently handled via Matlab. The CPU-based implementation is highly memory-efficient and provides considerable speed-up compared to the standard implementation not optimized for the mass-univariate approach. Further acceleration can be achieved on systems equipped with a CUDA-enabled GPU. Using the fastest GPU-based implementation, computation time for whole-brain estimates can be reduced from $9 \mathrm{~h}$ to $5 \mathrm{~min}$ in an exemplary data setting. Overall, the provided package facilitates the use of L1-regularization for fMRI activation analyses and enables an efficient employment of L1-regularization on whole-brain data and large sample sizes.
\end{abstract}

Keywords fMRI $\cdot$ L1-regularization $\cdot$ Lasso $\cdot$ Sparsity $\cdot$ Encoding model $\cdot$ GPU

\section{Introduction}

Over the last two decades, various fMRI activation analysis approaches have been established that involve a relatively large number of predictors. For example, single-trial models can be employed to obtain activation estimates for individual experimental trials (Mumford et al. 2012). The single-trial estimates can be subsequently used as input for further

Electronic supplementary material The online version of this article (https://doi.org/10.1007/s12021-020-09489-1) contains supplementary material, which is available to authorized users.

Holger Mohr

holger.mohr@tu-dresden.de

1 Department of Psychology, Technische Universität Dresden, 01062 Dresden, Germany analyses such as multivariate pattern analyses or connectivity analyses (Mumford et al. 2014; Rissman et al. 2004). As single-trial models require a separate predictor for each experimental trial, the resulting design matrices typically encompass a relatively large number of predictors.

The finite impulse response (FIR) model is another example for an fMRI activation analysis approach involving many predictors (Ollinger et al. 2001). In the FIR approach, the fMRI signal is modeled by a set of impulse response predictors, instead of a predefined hemodynamic response shape. This modeling approach can capture activation dynamics deviating from the canonical response shape, but typically involves a large number of predictors (growing proportionally with the size of the FIR basis set). Both in single-trial and FIR models, the large number of predictors can reduce the robustness of the beta estimates.

Another fMRI modeling approach typically involving a large number of predictors is the so-called encoding model 
approach (Naselaris et al. 2011; van Gerven 2017). In this approach, stimuli are represented in a high-dimensional feature space instead of being assigned to a low-dimensional set of categories. For example, instead of assigning visual stimuli to object categories such as houses, trees, etc., the stimuli are represented by a high-dimensional vector of feature weights. Such features may comprise a set of Gabor wavelets (Kay et al. 2008), may be extracted from deep neural networks (Güclü and van Gerven 2015) or may simply consist of pixel values (Schoenmakers et al. 2013). The encoding model approach has also been employed outside the visual domain, for example to characterize semantic representations of words (Huth et al. 2016). In this study, the feature space was defined as a basic dictionary of English words, and the model was fitted on whole-brain data. The high-dimensional representation of stimuli used in the encoding model approach typically translates into design matrices encompassing more predictors than time points. In this setting, a unique model fit can only be obtained by adding a regularization term to the model.

Model regularization adds additional constraints to the model fitting procedure on top of minimizing the error term. As the number of predictors approaches the number of collected time points (i.e. the length of the fMRI time series), model regularization becomes increasingly relevant, and for saturated models, regularization is indispensable. In the context of fMRI activation analyses, model regularization can improve the robustness of single-trial and FIR models, and is crucial for the encoding model approach.

For linear models, the two most common types of regularization are L1-regularization and L2-regularization (also known as lasso and ridge regression, Tibshirani 1996; Hoerl and Kennard 1970). While L1-regularization puts a threshold on the sum of absolute values of the beta estimates, L2regularization bounds the sum of squared beta-values. These two types of regularization can result in fundamentally different beta estimates: L2-regularized models return nonzero betavalues for all predictors, whereas L1-regularized models return a sparse model fit, that is, most of the beta-values are set to zero and only a few predictors are included in the model fit. Whether to employ L1- or L2-regularization depends on apriori assumptions on the data at hand. L2-regularization assumes that most of the predictors have an impact on the fMRI signal. In contrast, L1-regularization is based on the assumption that the fMRI signal can be modeled by a small fraction of the included predictors.

While both types of regularization have been employed in fMRI studies (Huth et al. 2016; Nishimoto et al. 2011), L2regularization seems to occur more frequently in the neuroimaging literature than L1-regularization. This might be partly explained by the fact that fitting L1-regularized models is considerably more expensive, in terms of computation time, than fitting L2-regularized or unregularized models. While L2-regularized and unregularized models can be estimated using closed-form solutions, L1-regularization requires an iterative fitting procedure. Thus, estimating L1-regularized models instead of L2- or unregularized models substantially increases the running time of fMRI analyses. For certain types of analyses, for example whole-brain analyses on large samples, it is virtually infeasible to employ L1-regularization.

Here, we aim to facilitate the estimation of L1-regularized models on fMRI data. In the following, we present a package of functions for estimating L1-regularized models that are optimized for the mass-univariate approach. We describe the implementation of the functions, how to set their parameters, and provide benchmark results for two exemplary data settings.

\section{Methods}

In the following, we assume to have an fMRI data matrix $Y$ of size $n \times v$, with $n$ being the number of time points and $v$ being the number of time series (e.g. the number of voxels). Moreover, we have a design matrix $X$ of size $n \times p$, with $p$ being the number of predictors. The beta-values are stored in a matrix $B$ of size $p \times v$. The intercept of the model is of size $n \times 1$ and is denoted $I$, and the intercept's beta-values are stored in $B^{0}$ of size $1 \times v$. Matrix columns are indexed by $j$. We assume that the columns of the design matrix $X$ are $\mathrm{z}$ scored, i.e. $\sum_{i=1}^{n} X_{i j}=0$ and $\frac{1}{n} \sum_{i=1}^{n} X_{i j}{ }^{2}=1$ for all $j=\{1$, $\cdots, p\}$. For a given regularization parameter $\lambda \geq 0$, the L1regularized model fit $\widehat{B}, \widehat{B}^{0}$ is expected to minimize, for each $j=\{1, \cdots, v\}$, the following objective function:

$\widehat{B}_{j}, \widehat{B}_{j}^{0}=\underset{B_{j}, B_{j}^{0}}{\operatorname{argmin}} \frac{1}{2 n}\left\|Y_{j}-X B_{j}-I B_{j}^{0}\right\|_{2}^{2}+\lambda\left\|B_{j}\right\|_{1}$

In contrast to L2-regularized or unregularized models, L1regularized model fits cannot be computed using a closedform solution. Instead, an iterative procedure is required to find $\widehat{B}$. In the following sections, we describe two different algorithms for fitting L1-regularized models, coordinate descent (Friedman et al. 2010) and alternating direction method of multipliers (ADMM, Boyd et al. 2010), and how we optimized and implemented these algorithms for mass-univariate analyses.

\section{The CPU-Based Implementation: lasso_mex}

In the CPU-based implementation, the model fit is computed using the coordinate descent algorithm proposed by Friedman et al. 2010. In each step of the iterative fitting procedure, the beta-value of a single predictor is updated while the remaining beta-values are fixed. As suggested by Friedman et al. 2010, 
the beta-values are computed using covariance updates, i.e. without explicitly computing residual values.

Friedman et al. 2010 suggest to compute covariances between predictors dynamically as required during the estimation process. However, in the mass-univariate setting, the same design matrix is used to model a large number of fMRI time series. Thus, instead of computing covariances between predictors dynamically for each voxel, we precompute the full covariance matrix of the design matrix before starting the coordinate descent, thereby avoiding redundant computations of predictor covariances across voxels.

Aside from precomputing the covariance matrix, our implementation includes features such as warm starts and active sets described in Friedman et al. 2010. The idea behind active sets is to iterate only through predictors whose beta-values were set to nonzero values at an earlier stage. Once convergence among the beta-values included in the active set is achieved, the algorithm iterates through all beta-values to check whether additional predictors have to be included. Using active sets can considerably speed up the estimation procedure, and moreover beta-values can be stored in sparse format, thereby reducing memory usage.

The estimation procedure for a given $\lambda$ parameter can be considerably accelerated by properly initializing the betavalues, instead of starting with all beta-values set to zero. Such an initialization can be obtained from a prior estimate using a larger $\lambda$ parameter. Generally, computation times increase as $\lambda$ becomes smaller, due to the larger number of nonzero beta-values. Thus, successively fitting models along a decreasing sequence of lambda parameters using warm starts is typically faster than starting from scratch for each lambda value (Friedman et al. 2010).

To further accelerate the estimation procedure, the CPUbased implementation distributes computations among multiple CPU cores using a parallel for-loop over the voxels, exploiting the fact that models are fitted independently across voxels in the mass-univariate analysis approach. To this end, the algorithm was implemented in C++ using OpenMP for parallelization. The performance improvement achieved by this parallelization step depends on the number of available CPU cores.

\section{How to Use lasso_mex}

While the underlying coordinate descent algorithm is implemented in $\mathrm{C}++$, the lasso mex function can be conveniently called from Matlab via the mex API. The function takes a design matrix $\mathrm{X}$, a matrix $\mathrm{Y}$ containing fMRI time series and a sequence of lambda-parameters lambda_seq as input. Technical parameters can be optionally specified using an options structure, otherwise default values are used. The columns of the design matrix X must be Z-scored, and X must not contain an intercept column. The function returns beta-values in sparse format, with $\mathrm{b}$-values containing the actual beta values, $b$ indexes containing the indexes of the values, and $\mathrm{N}$ nz containing the number of nonzero beta-values for each voxel. Unregularized intercept coefficients are returned in b0.

[b_values, b_indexes, N_nz, b0] = lasso_mex $(\mathrm{X}, \mathrm{Y}$, lambda_seq $)$;

The function lasso mex is written in Matlab and calls, after some sanity checks and precomputations, the MEX function lasso_mex_cpp.c, which is written in $\mathrm{C}++$ and runs the coordinate descent algorithm.

The resulting beta-values in sparse format can be converted to full format using the convert_betas_sparse_to_full function:

b_full $=$ convert_betas_sparse_to_full

$$
\text { (b_values, b_indexes, N_nz, size }(\mathrm{X}, 2) \text { ); }
$$

The sequence of lambda-parameters lambda_seq should be decreasing in order to benefit from warm starts as described above. Lambda-values are typically defined on a log-scale, e.g. $\lambda \in\left\{2^{k}, 2^{k-1}, \cdots, 2^{k-n}\right\}$. The lambda parameter determines the degree of model regularization. For lambda-values larger than a certain data-dependent threshold, all beta-values are set to zero, and the model fit only consists of the intercept. The critical threshold can be computed using the calculate_lambda_start function.

lambda_start $=$ calculate_lambda_start $(X, Y)$;

For lambda-values larger than lambda_start, all beta-values will be zero. Thus, the first value of the lambda sequence is typically set to lambda_start or to value from a predefined discrete scale that is close to lambda start. How to set the smallest value of the lambda-sequence is less clear and involves a trade-off between computation time and the desired degree of model saturation. Generally, estimating weakly regularized models requires longer computation times than estimating strongly regularized models. As the number of nonzero beta-values approaches the number of time points of the fMRI time series, model estimation can become timeconsuming and unstable in overparameterized settings (i.e. when $p>n$ ). Thus, the smallest value of the lambda sequence is typically chosen to achieve a certain degree of model saturation while keeping computation time within reasonable bounds.

Optionally, technical parameters of the lasso_mex function can be set via the options structure. The default values are:

options.n_iter_max $=1 \mathrm{e} 5$;

options. tol_value $=1 \mathrm{e}-3$;

options.buffer_factor $=3$; 
options. cpu_load_factor $=1$;

[b_values, b_indexes, N_nz, b0] = lasso_mex $(\mathrm{X}, \mathrm{Y}$, lambda_seq, options $)$;

The n_iter_max parameter defines an upper bound for the number of iterations performed by the coordinate descent algorithm. This parameter can be set to a larger value if the default value results in the error message Max. iter. Reached, no convergence!. However, reaching the maximum number of iterations can also indicate that model regularization is too weak and more stringent regularization is required.

The tol_value parameter determines the precision of the estimated beta-values. The coordinate descent algorithm stops when $\max _{j}\left|B_{j}^{\text {new }}-B_{j}^{\text {old }}\right|<$ tol_value. If higher than default precision is required, the tol_value parameter can be set to a smaller value, which will typically increase computation time. Vice versa, low-precision estimates can be obtained by setting tol_value to a larger value, which might accelerate the estimation procedure.

To minimize memory requirements, beta-values are stored in sparse format. The maximum number of nonzero betavalues per voxel is determined by the buffer_factor parameter. Internally, the buffer_factor is multiplied by $n$ (the number of rows of the design matrix $X$ ) in order to compute how much memory is preallocated for the beta-values. In well-defined settings, i.e. if $p \leq n$, the buffer factor parameter can be set to 1 . In overparameterized settings (i.e. if $p>n$ ), if the default value results in the error message $\mathrm{N}$ nz over maximum, larger buffer is required!, the buffer_factor parameter should be set to larger value. How the buffer factor impacts memory demands is detailed in the Supplementary Material section on memory usage.

The parameter cpu load factor determines the degree of CPU utilization. For a cpu_load_factor of 1 (default value), all CPU cores are engaged, whereas for a cpu_load_factor of 0 , only a single core is occupied. To distribute computations among all but one core, the cpu_load_factor can be set to 0.99 .

\section{The GPU-Based Implementations: lasso_mexcuda and lasso_GPU}

The two GPU-based implementations are based on the alternating direction method of multipliers (ADMM) algorithm described in Boyd et al. 2010. In contrast to the coordinate descent algorithm, the ADMM algorithm does not sequentially cycle trough the beta coefficients but instead all beta coefficients are updated simultaneously via matrix multiplication. While the ADMM procedure is less memory-efficient than coordinate descent, it can be accelerated on the GPU by parallelizing matrix multiplications and other steps. The first version (lasso_mexcuda) can be called from Matlab and does not require any Matlab toolboxes. After some sanity checks and precomputations, the mex functions ADMMcublasOverMex.c or ADMMcublasUnderMex.c are called (depending on whether the design matrix is overparameterized or not), which are written in $\mathrm{C}++$. These functions then call ADMMcublasOver.cu or ADMMcublasUnder.cu, which contain CUDA code calling functions from the cuBLAS library to run the ADMM algorithm on the GPU. The second version (lasso_gpu) is implemented directly in Matlab using gpuArray and thus depends on the Parallel Computing Toolbox. For both versions, a CUDA-enabled GPU device is required (see also Table 1).

Both lasso_mexcuda and lasso_gpu use warm starts along the supplied lambda sequence, and the same considerations on the choice of the lambda sequence as discussed above in the lasso_mex section also apply to the lasso_mexcuda and lasso_gpu functions.

\section{How to Use lasso_mexcuda and lasso_gpu}

The functions take a design matrix $\mathrm{X}$, a matrix $\mathrm{Y}$ containing fMRI time series and a sequence of lambda-parameters lambda_seq as input. Technical parameters can be optionally specified using an options structure, otherwise default values are used. The columns of the design matrix $\mathrm{X}$ must be $\mathrm{z}$ scored, and $\mathrm{X}$ must not contain an intercept column. Betavalues are returned in full format in $\mathrm{B}$, and unregularized intercept coefficients are returned in B0. As lasso_mexcuda and lasso_gpu are optimized for the GPU, B and B0 are returned in single-precision format.

$[\mathrm{B}, \mathrm{B} 0]=$ lasso $\_$mexcuda $(\mathrm{X}, \mathrm{Y}$, lambda_seq $)$;

$[\mathrm{B}, \mathrm{B} 0]=$ lasso_gpu $(\mathrm{X}, \mathrm{Y}$, lambda_seq $)$;

Optionally, the following technical parameters can be specified (set to default values here):

options.n_iter_max $=1 \mathrm{e} 5$;

options.tol_value $=1 \mathrm{e}^{-3}$;

options.buffer_size $=8192$;

$[\mathrm{B}, \mathrm{B} 0]=$ lasso_mexcuda $(\mathrm{X}, \mathrm{Y}$, lambda_seq, options $)$;

$[\mathrm{B}, \mathrm{B} 0]=$ lasso_gpu $(\mathrm{X}, \mathrm{Y}$, lambda_seq, options $)$; 
Table 1 Overview over hardware and software requirements of the different functions for estimating L1-regularized linear models. The lasso function is part of the Statistics and Machine Learning Toolbox and only included here for benchmarking purposes (see main text). The lasso_mex, lasso_mexcuda and lasso_gpu functions introduced here are specifically optimized for the mass-univariate analysis approach and depend on different hardware and software configurations

\begin{tabular}{|c|c|c|c|c|}
\hline Function & Hardware requirements & Software requirements & Algorithm & Implementation \\
\hline lasso & CPU & Matlab, Statistics and ML Toolbox & Coordinate descent & Matlab \\
\hline lasso_mex & CPU & Matlab & Coordinate descent & $\mathrm{C}++$, OpenMP \\
\hline lasso_mexcuda & CPU + CUDA-enabled GPU & Matlab, CUDA Toolkit & ADMM & $\mathrm{C}++$, cuBLAS \\
\hline lasso_gpu & CPU + CUDA-enabled GPU & Matlab, Parallel Computing Toolbox & ADMM & Matlab \\
\hline
\end{tabular}

The $\mathrm{n}$ iter max parameter defines an upper bound for the number of iterations performed by the ADMM algorithm. This parameter can be set to a larger value if the default value results in the error message Max. iter. Reached, no convergence!. However, reaching the maximum number of iterations can also indicate that model regularization is too weak and more stringent regularization is required.

The tol value parameter determines the precision of the estimated beta-values. If higher than default precision is required, the tol_value parameter can be set to a smaller value, which will typically increase computation time. Vice versa, low-precision estimates can be obtained by setting tol_value to a larger value, which might accelerate the estimation procedure.

The buffer_size determines how many voxels are simultaneously processed on the GPU. Setting this parameter to a larger value might accelerate the estimation procedure, provided that the GPU has sufficient memory resources. If buffer_size exceeds the memory capacity of the GPU, Matlab terminates the function call with an error message. Memory requirements can be estimated based on the heuristics provided in the Supplementary Material section on memory usage.

\section{Benchmarking}

The three functions lasso_mex, lasso_mexcuda and lasso_gpu were benchmarked in two data settings to demonstrate the efficiency of the implementations. In both benchmarks, $X$ and $Y$ data were randomly drawn from the normal distribution. Benchmark A corresponds to an overparameterized model, representing for example an encoding model with a large feature space. The number of time points was set to $n=300$, which corresponds to a 10 min scanner run for a TR of $2 \mathrm{~s}$. The number of predictors was set to $p=5000$, i.e. $p \gg n$. The model was estimated on $v=65536$ voxels, approximately corresponding to whole-brain data for an isotropic $3 \mathrm{~mm}$ resolution. The lambda sequence was set to $\left\{2^{-2}, 2^{-3}, \cdots, 2^{-6}\right\}$. Benchmark B corresponds to a well-defined setting, e.g. a single-trial or FIR model. The number of time points and voxels in setting $B$ were identical to setting $A$, but the number of predictors was set to $p=200$, i.e. $p<n$. The models were estimated for a single lambda-value $\lambda=2^{-4}$ to allow for a comparison of the computation times with L2-regularized and unregularized model fits.

The three functions lasso_mex, lasso_mexcuda and lasso_gpu were compared to the lasso function that is part of Matlab's Statistics and Machine Learning Toolbox. The lasso function was repeatedly called to fit models for all voxels using a for-loop, taking a single time series as input in each call.

The benchmarks were run on a workstation equipped with a dual Intel Xeon E5-2665 CPU (16 cores overall), 32 GB memory and an Nvidia Quadro P2000 GPU. The functions were benchmarked on Windows 10 64-bit and Matlab R2019b, as well as Ubuntu 18.04 LTS and Matlab R2018b. On Windows, lasso_mex was compiled using mex and the MSVC compiler of Visual Studio 2017, and lasso_mexcuda using CUDA Toolkit 10.1.243. On Linux, lasso_mex was compiled using mex and gec 6.5, and lasso_mexcuda using CUDA Toolkit 9.1.85.

Moreover, to assess the impact of the GPU hardware, we compared two different GPU devices using benchmark A, Nvidia's Quadro P2000 (1024 cores, 5 GB memory) and Tesla V100 SXM2 (5120 cores, 16 GB memory). To this end, the functions lasso_mexcuda and lasso_gpu were run on a p3.2xlarge instance on Amazon Web Services (AWS) using a Matlab Amazon Machine Image (AMI).

\section{Code and Software Requirements}

The presented software package including compiled binaries and source code is available at https:/git.io/JvUpi. Data input/ output is handled via Matlab for all functions of the package. The functions have been tested using Matlab R2019b and R2018b, but should work with other versions as well. Moreover, the GPU-accelerated functions require either the CUDA toolkit (lasso_mexcuda) or Matlab's Parallel Computing Toolbox (lasso_gpu). Note that each Matlab version requires a specific version of the CUDA toolkit, as listed here: https://mathworks.com/help/parallel-computing/gpusupport-by-release.html. GPU devices should have compute capability $>=3.0$. 


\section{Results}

On both benchmarks A and B, the lasso mex function was considerably faster than the standard lasso function using a single CPU core, showing that the coordinate descent algorithm underlying lasso_mex is efficiently implemented (see Fig. 1). Setting the lasso_mex function to distribute computations across multiple $\bar{C}$ PU cores led to further reductions of computation time. More details on how computation time varied as a function of CPU cores are given in Supplementary Fig. 1. The parallel implementation of the ADMM algorithm on the GPU (lasso_mexcuda, lasso_gpu) provided further acceleration for benchmark A, with lasso_gpu being considerably faster than lasso_mexcuda. In absolute numbers, a reduction of computation time from approximately $9 \mathrm{~h}$ to $5 \mathrm{~min}$ could be achieved on Windows using the lasso_gpu function, see Supplementary Table 1. As shown in benchmark B, L2regularized (ridge regression) or unregularized (ordinary least squares, OLS) model estimation remains faster than accelerated L1-regularization.

The comparison of two different GPU devices using benchmark A on Linux revealed that the larger device (Tesla V100) achieves a $4.5 \mathrm{x}$ speed-up over the smaller card (Quadro P2000), see Fig. 2. This acceleration approximately corresponds to the ratio of available CUDA cores of 5120:1024, see Supplementary Fig. 2 for more details. In absolute numbers, computation time could be further reduced to $1 \mathrm{~min}$ using the lasso_gpu function on the V100 device, see Supplementary Table 2.
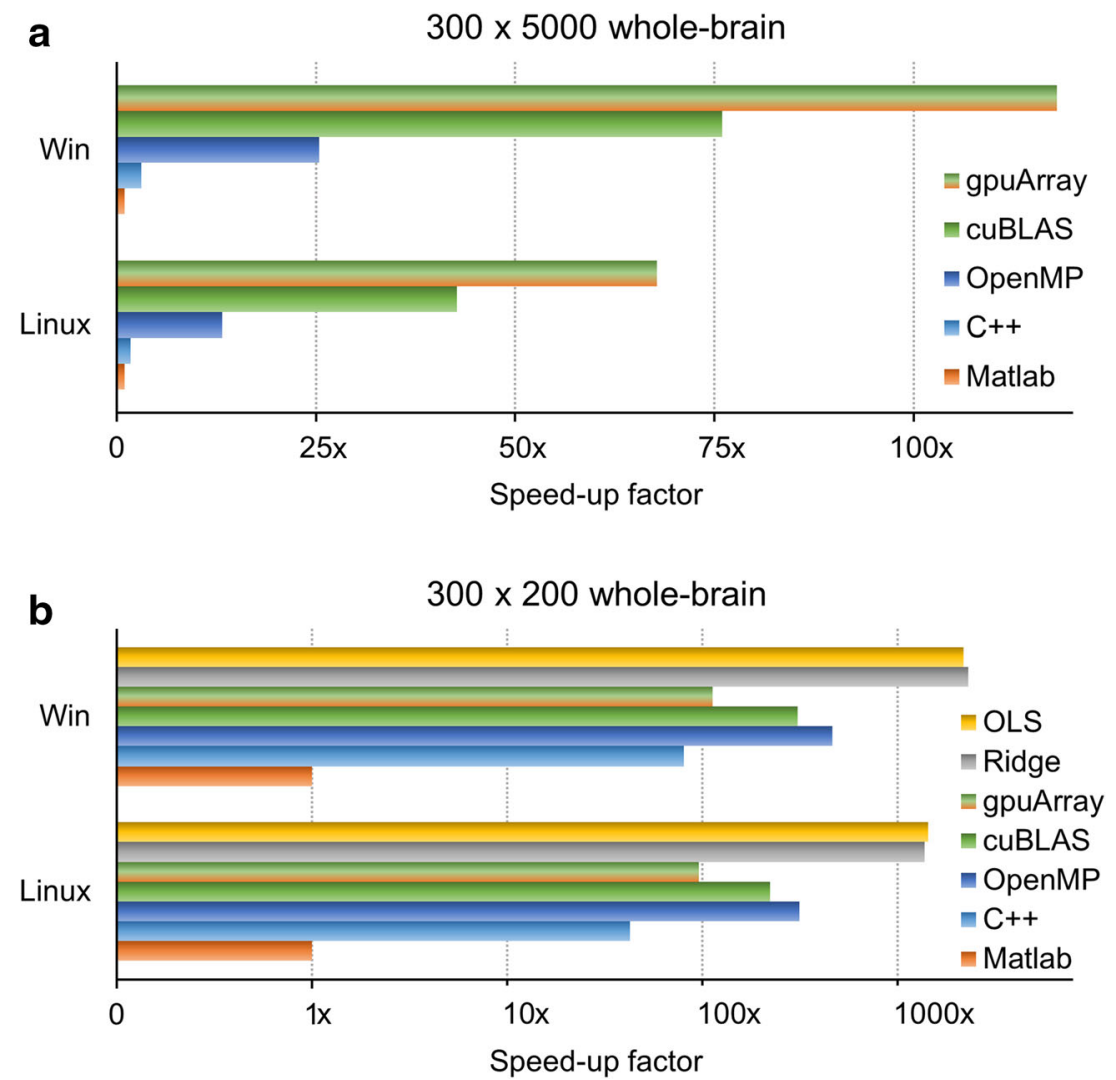

Fig. 1 Benchmark results for the three lasso functions lasso_mex, lasso mexcuda and lasso_gpu. (a): Benchmark for an overparameterized setting with $n=300$ time points and $p=5000$ predictors, corresponding for example to an encoding model with a large feature space. The lasso mex function required considerably less running time for whole-brain estimates than the standard lasso function (Matlab, orange) on a single CPU core $(\mathrm{C}++$, light blue). Distributing computations across multiple CPU cores further reduced the running time of the lasso_mex function (OpenMP, dark blue). The lasso mexcuda function, which runs the ADMM algorithm on a GPU using the cuBLAS library, further accelerated the estimation procedure (cuBLAS, green). The lasso_gpu function, which runs the ADMM algorithm on the GPU using the Parallel Computing Toolbox, provided the highest speed-up factor for benchmark
A (gpuArray, green/orange). (b): Benchmark for a well-defined setting with fewer predictors than time points $(n=300, p=200)$, corresponding for example to a single-trial or FIR model. Again, on a single CPU core, the lasso_mex function fitted L1-regularized models more efficiently than the standard lasso function. Further speed-up could be achieved by distributing computations across multiple CPU cores. The GPU-based implementations (lasso_mexcuda, lasso_gpu) performed not as fast as the multicore CPU version on this benchmark, as the GPU was not fully occupied in this small-scale setting. Unregularized (OLS, yellow) or L2regularized (ridge, gray) model estimation using closed-form solutions remains faster than accelerated L1-regularization. Absolute computation times are given in Supplementary Table 1 
Fig. 2 Benchmark results for two different GPU devices. Nvidia's Tesla V100 GPU device yielded a speed-up of approximately $4.5 \mathrm{x}$ over the Quadro P2000 card, which roughly corresponds to the ratio of 5120:1024 cores.

Absolute computation times are given in Supplementary Table 2
$300 \times 5000$ whole-brain

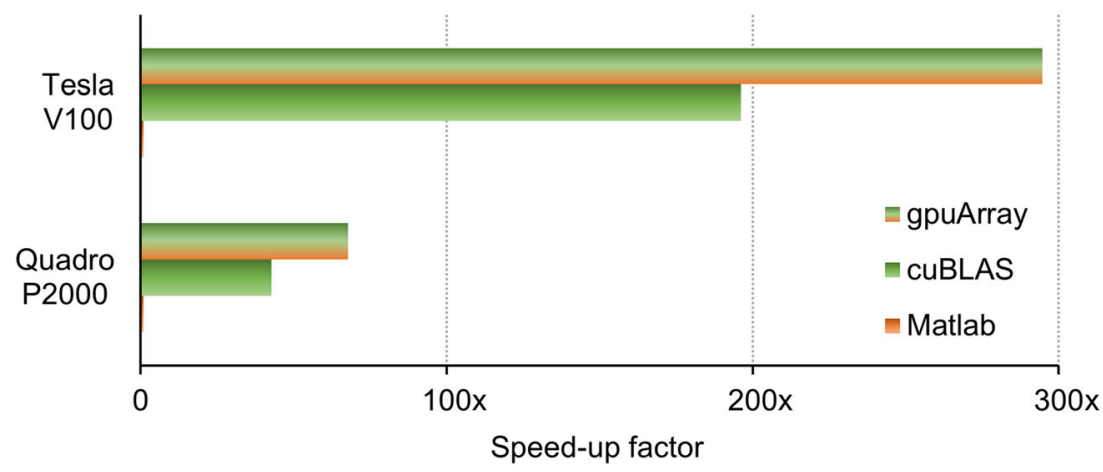

\section{Discussion}

We introduced a package of functions for estimating L1regularized models in the mass-univariate fMRI analysis approach. The presented functions significantly accelerate the model estimation procedure and thereby facilitate the use of L1-regularization in the mass-univariate approach and enable its application on whole-brain data and large samples. While the presented benchmarks were performed on data corresponding to a single fMRI scanning session, efficiency gains scale up linearly for multiple sessions per subject and multiple subjects per sample. For example, for a sample of 20 subjects and 5 scanning sessions per subject, a computation time reduction from $9 \mathrm{~h}$ to $5 \mathrm{~min}$ per scanning session translates into a reduction from 37 days to $8 \mathrm{~h}$ for the whole sample.

This speed-up makes it practically feasible to employ L1regularization in the context of the encoding model approach. While the majority of studies using the encoding model approach have been focused on visual processing (van Gerven 2017), Huth et al. 2016 have shown that an extension to other domains such as whole-brain semantic representations is possible and promising. Data-driven generation of predictive features, for example via deep neural networks (Güclü and van Gerven 2015; Kell et al. 2018; Mohr et al. 2019), typically results in large feature spaces and therefore requires efficient model estimation procedures. Given the rapid advancement of machine learning in a range of domains that are also relevant for neuroimaging (e.g. geometric representations of objects (Eslami et al. 2018) or spatial navigation (Banino et al. 2018)), we expect a proliferation of the encoding model approach for predicting fMRI signals via machine-learning generated features. Estimating such large-scale encoding models using L1-regularization can be efficiently performed by the functions presented here.

Moreover, the presented functions facilitate the use of L1regularization for modeling approaches such as single-trial and FIR models (Mumford et al. 2012; Ollinger et al. 2001). To our knowledge, the impact of L1-regularization has not yet been systematically evaluated for these types of models. Using the functions presented here, future studies can efficiently evaluate how L1-regularization impacts the robustness and predictiveness of single-trial and FIR models in comparison to L2-regularization or unregularized model estimation.

In the following, we discuss some limitations of L1regularization in general and the specific implementations presented here. It is important to note that the beta estimates returned by L1-regularized models are typically sparse and thus not normally distributed. Depending on how the beta estimates are used in subsequent analysis steps, a modification of some of these steps might be required. For example, for aggregating data, median values can be used instead of arithmetic means to preserve sparsity. Moreover, when using L1regularization for example for estimating single-trial models, both the spatial activations patterns and the beta-series are typically sparse. To compute correlations between such sparse spatial patterns or beta-series, rank-based correlation measures should be used instead of Pearson correlations. In contrast, using sparse beta estimates for predicting fMRI signals in the context of the encoding models approach would typically not require specific adjustments. For example, to compute model predictions of an L1-regularized model, the design matrix of a given test dataset can simply be multiplied with the sparse beta estimates obtained on a training dataset. With respect to decoding techniques such as multivariate pattern analysis (MVPA), it should be noted that although it would be technically possible to use the presented functions for estimating decoding models (by filling the design matrix with fMRI data), no acceleration can be expected in this case, as decoding models are typically estimated for a single outcome variable only. Since the presented functions are optimized for the mass-univariate approach, estimation procedures are only accelerated in settings involving many outcome variables.

In conclusion, the functions introduced here significantly accelerate L1-regularization in the mass-univariate setting and make it practically feasible to estimate L1-regularized models on whole-brain data and large samples. 
Information Sharing Statement The presented software package including compiled binaries and source code is available at https://git.io/JvUpi.

Acknowledgments This work was supported by the German Research Foundation (DFG), grant CRC 940, project Z2. We thank the Center for Information Services and High Performance Computing (ZIH) at TU Dresden for generously providing computing resources.

\section{Availability of Data and Material Not applicable.}

Code Availability The presented software package including compiled binaries and source code is available at https:/git.io/JvUpi.

Funding Open Access funding provided by Projekt DEAL. This work was supported by the German Research Foundation (DFG), grant CRC 940, project Z2. We thank the Center for Information Services and High Performance Computing ( $\mathrm{ZIH})$ at TU Dresden for generously providing computing resources.

\section{Compliance with Ethical Standards}

Conflicts of Interest/Competing Interests The authors declare that they have no conflict of interest.

Open Access This article is licensed under a Creative Commons Attribution 4.0 International License, which permits use, sharing, adaptation, distribution and reproduction in any medium or format, as long as you give appropriate credit to the original author(s) and the source, provide a link to the Creative Commons licence, and indicate if changes were made. The images or other third party material in this article are included in the article's Creative Commons licence, unless indicated otherwise in a credit line to the material. If material is not included in the article's Creative Commons licence and your intended use is not permitted by statutory regulation or exceeds the permitted use, you will need to obtain permission directly from the copyright holder. To view a copy of this licence, visit http://creativecommons.org/licenses/by/4.0/.

\section{References}

Banino, A., Barry, C., Uria, B., Blundell, C., Lillicrap, T., Mirowski, P., Pritzel, A., Chadwick, M. J., Degris, T., Modayil, J., Wayne, G., Soyer, H., Viola, F., Zhang, B., Goroshin, R., Rabinowitz, N., Pascanu, R., Beattie, C., Petersen, S., Sadik, A., Gaffney, S., King, H., Kavukcuoglu, K., Hassabis, D., Hadsell, R., \& Kumaran, D. (2018). Vector-based navigation using grid-like representations in artificial agents. Nature, 557, 429-433.

Boyd, S., Parikh, N., Chu, E., Peleato, B., \& Eckstein, J. (2010). Distributed optimization and statistical learning via the alternating direction method of multipliers. Found. Trends® Mach. Learn., 3, $1-122$.
Eslami, S. M. A., Jimenez Rezende, D., Besse, F., Viola, F., Morcos, A. S., Garnelo, M., Ruderman, A., Rusu, A. A., Danihelka, I., Gregor, K., Reichert, D. P., Buesing, L., Weber, T., Vinyals, O., Rosenbaum, D., Rabinowitz, N., King, H., Hillier, C., Botvinick, M., Wierstra, D., Kavukcuoglu, K., \& Hassabis, D. (2018). Neural scene representation and rendering. Science, 360, 1204-1210.

Friedman, J. H., Hastie, T., \& Tibshirani, R. (2010). Regularization paths for generalized linear models via coordinate descent. J Stat Softw, $33,1-22$.

Güclü, U., \& van Gerven, M. A. J. (2015). Deep neural networks reveal a gradient in the complexity of neural representations across the ventral stream. J Neurosci, 35, 10005-10014.

Hoerl, A. E., \& Kennard, R. W. (1970). Ridge regression: Biased estimation for nonorthogonal problems. Technometrics, 42, 80-86.

Huth, A. G., de Heer, W. A., Griffiths, T. L., Theunissen, F. E., \& Gallant, J. L. (2016). Natural speech reveals the semantic maps that tile human cerebral cortex. Nature, 532, 453-458.

Kay, K. N., Naselaris, T., Prenger, R. J., \& Gallant, J. L. (2008). Identifying natural images from human brain activity. Nature, 452, $352-355$.

Kell, A. J. E., Yamins, D. L. K., Shook, E. N., Norman-Haignere, S. V., \& McDermott, J. H. (2018). A task-optimized neural network replicates human auditory behavior, predicts brain responses, and reveals a cortical processing hierarchy. Neuron, 98, 630-644 e16.

Mohr, H., Cichy, R, M. \& Ruge, H. (2019). Deep neural networks can predict human behavior in arcade games. Proceedings of the 2019 conference on cognitive computational neuroscience, Berlin, Germany. DOI: https://doi.org/10.32470/CCN.2019.1043-0

Mumford, J. A., Turner, B. O., Ashby, F. G., \& Poldrack, R. A. (2012). Deconvolving BOLD activation in event-related designs for multivoxel pattern classification analyses. NeuroImage, 59, 26362643.

Mumford, J. A., Davis, T., \& Poldrack, R. A. (2014). The impact of study design on pattern estimation for single-trial multivariate pattern analysis. NeuroImage, 103, 130-138.

Naselaris, T., Kay, K. N., Nishimoto, S., \& Gallant, J. L. (2011) Encoding and decoding in fMRI. Neurolmage, 56, 400-410.

Nishimoto, S., Vu, A. T., Naselaris, T., Benjamini, Y., Yu, B., \& Gallant, J. L. (2011). Reconstructing visual experiences from brain activity evoked by natural movies. Curr Biol, 21, 1641-1646.

Ollinger, J. M., Shulman, G. L., \& Corbetta, M. (2001). Separating processes within a trial in event-related functional MRI. NeuroImage, $13,210-217$.

Rissman, J., Gazzaley, A., \& D’Esposito, M. (2004). Measuring functional connectivity during distinct stages of a cognitive task. NeuroImage, 23, 752-763.

Schoenmakers, S., Barth, M., Heskes, T., \& van Gerven, M. (2013). Linear reconstruction of perceived images from human brain activity. NeuroImage, 83, 951-961.

Tibshirani, R. (1996). Regression shrinkage and selection via the lasso. $J$ $R$ Stat Soc Ser B Methodol, 58, 267-288.

van Gerven, M. A. J. (2017). A primer on encoding models in sensory neuroscience. J Math Psychol, 76, 172-183.

Publisher's Note Springer Nature remains neutral with regard to jurisdictional claims in published maps and institutional affiliations. 